\title{
Realidad de la Educación Física en la Escuela Rural: una Revisión Sistemática Reality of Physical Education in Rural Schools: a Systematic Review
}

*Sergio Ferrando Felix, **Oscar Chiva-Bartoll, *Carmen Peiró-Velert

*Universidad de Valencia (España), **Universidad Jaume I (España)

Resumen. Esta revisión pretende analizar la investigación publicada a nivel nacional e internacional en el ámbito de la Educación física en el contexto educativo rural. M: El procedimiento de investigación se centró en la revisión de los artículos publicados en las bases de datos WoS, Scopus, ERIC, Sportdiscus y Dialnet. Los artículos contaron con los siguientes criterios de inclusión y exclusión para asegurar la relevancia y el ajuste temático de la revisión: área de conocimiento, fecha de publicación, idioma y tipo de documento. Además, se combinaron diferentes términos de búsqueda vinculados con el campo de estudio: rural school, physical education and sport. Tras aplicar los criterios de selección, se analizaron las 62 publicaciones resultantes. R y D: La Educación Física en la escuela rural es un tema de investigación olvidado a nivel internacional. Los escasos estudios se vinculan a la mejora de la salud y del bienestar de una sociedad considerada con menores recursos y apenas se encuentran referencias sobre cuestiones educativas. En la escena nacional, todos los estudios examinados presentan análisis teórico-descriptivos y algunos relatan experiencias prácticas. Esta falta de estudios evidencia la necesidad de realizar investigaciones que indaguen en las potencialidades educativas de la Educación física en la escuela rural.

Palabras clave: educación física, escuela rural, deporte, contextos desfavorecidos y revisión sistemática.

\begin{abstract}
This review aims to analyze research published nationally and internationally in the field of Physical education in rural educational contexts. M: The research procedure focused on the review of articles published in WoS, Scopus, ERIC, Sportdiscus, and Dialnet databases. In order to ensure the relevance and thematic adjustment of the reviewed articles, the following inclusion and exclusion criteria were followed: area of knowledge, date of publication, language, and type of document. In addition, different search terms linked to the field of study were combined: rural school, physical education, and sport. After applying the selection criteria, 62 resulting publications were analyzed. R and D: Physical Education in rural schools is an often disregarded research topic at the international level. The few existing studies are linked to improving health and well-being of a society considered to have fewer resources, whilst there references to educational issues are scarce. In the national scene, all the studies examined presented theoretical-descriptive analysis, only some referring to practical experiences. This lack of studies emphasizes the need to carry out research investigating the educational potential of Physical education in rural schools.
\end{abstract}

Keywords: Physical education, rural school, sport, disadvantaged contexts and systematic review.

\section{Introducción}

La escuela rural se presenta como una realidad a tener en cuenta en el debate educativo actual ya que, pese a estar inmersos en un proceso de globalización pedagógica, los entornos locales emergen como una fuente con grandes posibilidades educativas. El propio concepto engloba una gran complejidad y diversidad terminológica, que se explica a partir de diversos criterios geográficos, sociológicos, demográficos y pedagógicos (Bustos, 2009; Domingo, 2014; Feu; 2004; Santamaria, 1996). Estos criterios nos permiten definir la escuela rural como aquella que se encuentra situada en poblaciones pequeñas, de entre 1000 y 2000 habitantes aproximadamente, ubicadas en espacios rurales o de tradición rural. Es decir, en las zonas que siguen siendo rurales y en aquellas en las que, pese a la reconversión de los pueblos debida a la proximidad de las grandes ciudades y a cambios en los sectores de producción, siguen manteniendo la cultura del entorno rural. Por otra parte, la escuela rural también puede ser entendida en la actualidad como una escuela única que actúa como foco cultural central de una población (Bustos, 2009; Domingo, 2014). Atendiendo a su gran diversidad, las escuelas rurales pueden presentar una estructura organizativa heterogénea, con una configuración pedagógica y didáctica multidimensional orientada a promover la innovación educativa y el desarrollo de la comunidad

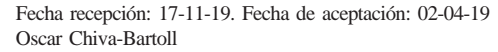

rural a la que pertenece (Boix, 1995; Santamaria, 1996). En cualquier caso, tradicionalmente la escuela rural se ha visto condicionada por una gran cantidad de prejuicios sociales que han afectado a la imagen que hoy en día se tiene de ella. Se trata, por desgracia, de una institución que siempre ha tenido que convivir en un segundo plano por detrás del modelo estándar ordinario, sin que por tanto se tuviera en cuenta su propia realidad, la del mundo rural (Bustos, 2011).

Lo mismo ha sucedido con el otro eje vertebrador de la presente revisión, la Educación Física (EF). Nos referimos a un área curricular que ha sido menospreciada históricamente debido a su carácter motriz y eminentemente práctico, lo que cuestionaba sus posibilidades de aprendizaje cognitivo y, en consecuencia, generaba falta de interés social (Hirst, 1974; Hirst \& Peters, 1970; García-Puchades \& Chiva-Bartoll, 2018). En cambio, todo indica que esta materia ya trabajaba con muchos de aquellos principios que actualmente se plantean hoy como necesarios para llevar a cabo una educación de calidad, como la variedad de estilos de enseñanza para atender a la diversidad, el cambio de rol del profesorado y del alumnado, el uso de las prácticas educativas, etc. (Blázquez, 2016; Devís-Devís, 2018; Kirk, 2010).

Así pues, si combinamos un modelo escolar tradicionalmente menospreciado y una materia curricular denostada socialmente, todo hace pensar que el enfoque de la EF en la escuela rural haya sido poco acertado. Por ello conviene hacer un análisis que nos ayude a conocer si es así, pues teóricamente todo apunta a que su combinación puede ser muy positiva. Sobre todo, porque combinar EF y escuela rural supone plantear un modelo educativo abierto, comprometido con la comunidad, que valora la cultura en que se 
encuentra inmerso, la diversidad, los diferentes ritmos de aprendizaje, el entorno propio y su alumnado, para garantizar una enseñanza que respete la identidad de cada uno (Sepúlveda y Gallardo, 2011). Por este motivo, «la escuela rural tiene futuro porque todavía existe, no es una reliquia del pasado, es un hecho real, actual y muy difundido en la geografía española» (Abós, 2007, p.230). Será en este momento, al hablar de los beneficios pedagógicos de la escuela rural, cuando el modelo educativo que promueve permita visibilizar todo su potencial en el área de EF como uno de sus ejes vertebradores. Sobre todo, porque la EF permite a la escuela rural trabajar en el medio natural, hacer uso de materiales alternativos de elaboración propia, vincular los contenidos curriculares con la cultura de la comunidad o trabajar de forma más individualizada, familiar y abierta a las familias (López-Pastor, 2008; Ruiz-Omecaña, 2008).

En este sentido, surge la necesidad de llevar a cabo esta revisión con el objetivo de descubrir si esta visión social denostada sobre el contexto rural y su tratamiento en el área de EF sigue presente en los estudios a nivel nacional e internacional.

\section{Método}

\section{Estrategia de búsqueda}

La búsqueda en las bases de datos, la filtración y la selección de los artículos, así como el análisis y la posterior redacción de los resultados del presente texto, ha sido desarrollada por un grupo de tres investigadores, cuyas líneas de trabajo se centran en la teoría de la educación, la didáctica de la EF, los métodos pedagógicos y las actuaciones en poblaciones vulneradas.

Para llevar a cabo este estudio de manera sistemática y rigurosa se han seguido los pasos propuestos por SenaGomes \& De Oliveira-Caminha (2014). Una vez identificada la necesidad de efectuar la presente revisión bibliográfica, se optó por llevar a cabo una búsqueda en cinco bases de datos. En primer lugar, se utilizaron aquellas con mayor reconocimiento a nivel internacional como son Web of Science (WoS) y Scopus-Elvesier. En segundo lugar, se consultaron las bases de datos específicas sobre los campos de conocimiento en los que se centra la revisión. Por un lado, Education Resources Information Center (en adelante ERIC), ya que está considerada una base de datos de gran relevancia en el campo de la educación y la pedagogía. Por otra parte, Sportdiscus, una de las bases de datos con mayor tradición en el campo de la EF y deportiva. Finalmente, se incorpora en el análisis una base de datos local como Dialnet, debido a que uno de los principales ejes de esta investigación, el modelo educativo de la escuela rural, depende en gran medida del contexto de referencia en el que se desarrolla.

La búsqueda se desarrolló entre los meses de marzo y diciembre de 2017. Se tomaron aquellas publicaciones que relacionaban la escuela rural con la EF y el deporte. Para ello se desarrolló de manera sistemática una búsqueda que combinara, mediante el boleano «and», términos de los dos campos anteriormente establecidos. Para el primer campo se utilizó tanto la terminología inglesa «rural school» como la española «escuela rural» 0 «escuela en el medio rural». Para el segundo campo, se utilizaron los términos «physical education» y «sport», dada la consideración en algunos países anglosajones del deporte como sinónimo de EF. Asimismo, en español se utilizaron los términos «educación física» y «deporte».

El procedimiento de investigación se caracterizó por una primera fase heurística basada en la recopilación de diversas fuentes de información surgidas a partir de los términos y sus respectivas combinaciones. Estas debían aparecer en el título, en las palabras clave o en el resumen de las diferentes bases de datos. A continuación, se procedió a una segunda fase de filtrado en la que se procedió a especificar la búsqueda según la finalidad del estudio, eliminando información perteneciente a otros campos de estudio (atendiendo a los criterios de inclusión y exclusión expuestos en el siguiente apartado). Finalmente, fue llevada a cabo una fase hermenéutica en la que analizamos el contenido de las diversas fuentes, para clasificarlas valorando la importancia dentro de nuestro análisis.

\section{Criterios de inclusión y exclusión}

Los criterios de inclusión y exclusión utilizados permitieron eliminar aquella información vinculada con la terminología empleada pero ajena a los objetivos del estudio, dotando a la revisión de una mayor rigurosidad.

En primer lugar, se acotaron los idiomas en los que estaban escritas las publicaciones a analizar, optando por el inglés y el español a fin de evitar textos ininteligibles para el equipo investigador.

En segundo lugar, se determinó el intervalo de años que comprende la revisión, comprendido entre 2000 y 2017 (ambos incluidos). Situamos el inicio en esta fecha porque muchos de los estudios llevados a cabo a lo largo del siglo XX se encuentran obsoletos debido al avance y mejora de las escuelas rurales en la actualidad, así como de los métodos pedagógicos que se están llevando a cabo. Por otro lado, debemos exponer que han sido analizados todos los estudios hasta 2017.

En tercer lugar, siguiendo criterios de actualización e impacto, se ha utilizado el filtro del «tipo de documento» 0 document type, limitando la búsqueda a artículos, libros o capítulos de libro, y excluyendo otros documentos como actas de congresos o tesis doctorales.

En cuarto y último lugar, se han filtrado los resultados en función del «área de conocimiento» o «subject area» ya que, como producto de una criba inicial, se obtuvieron multitud de resultados vinculados con las ciencias de la salud que no se ajustaban a los objetivos del presente estudio. Por tanto, se decidió optar por aquellas áreas de conocimiento vinculadas con la educación y las ciencias sociales en aquellas bases de datos en las que esta selección era posible (ERIC y Sportdiscus no disponen de ella). Es decir, en WoS y Scopus se marcaron las opciones Social Sciences y Arts and Humanities. Finalmente, en Dialnet se marcó la selección de los artículos vinculados con «psicología y educación» y «ciencias sociales».

Los textos que superaron el filtrado llevado a cabo en las bases de datos fueron clasificados por el equipo de investigadores atendiendo a diferentes parámetros: idioma, temática que se trabaja, nivel educativo, método de estudio, contextualización, año de publicación y revista de proceden- 
cia. Esta distinción sirvió para eliminar aquellos artículos que, a pesar de haber superado la criba del filtrado, no hablaran específicamente de los dos temas en los que se centra la revisión: escuelas rurales y EF, actividad física o deporte. La figura 1 refleja gráficamente el procedimiento seguido.

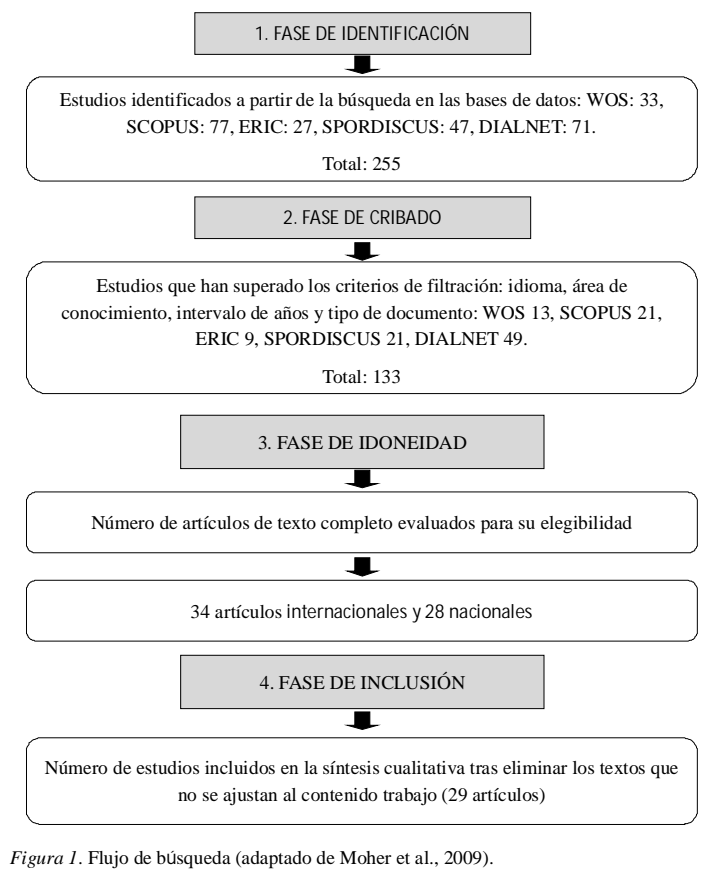

\section{Resultados}

\section{Publicaciones extraídas de las diferentes bases de da- tos \\ Con la búsqueda inicial se accedió a un total de 115 pu-} blicaciones, de las cuales 46 eran en inglés y 69 en español, número que se redujo a 62 trabajos resultantes al aplicar los criterios de inclusión y exclusión enunciados, así como el posterior análisis de significado. La tabla 1 recoge las publicaciones definitivas y los resultados obtenidos.

Tal y como indica la misma, al combinar los términos «escuela rural» $\mathrm{y}$ «EF» en español encontramos un total de 28 publicaciones (de las 46 encontradas en la búsqueda inicial) que provenían prácticamente en su totalidad de la base de datos española Dialnet.

Al llevar a cabo esta misma combinación con la terminología inglesa «rural school» and "physical education», se encontraron entre 21 y 35 trabajos en función de la base de datos utilizada. Sin embargo, al aplicar los criterios de filtrado y eliminar las repeticiones de artículos, la totalidad fue de 22 publicaciones.

Por su parte, al combinar en español «escuela rural» y «deporte» los resultados fueron inicialmente 26, provenientes de Dialnet. Sin embargo, al aplicar los filtros la búsqueda se redujo a 19 y al analizar el contenido todos ellos fueron descartados porque ya aparecían en la búsqueda anterior que combinaba escuela rural y EF. Finalmente, al combinar en inglés «rural school» and «sport» los resultados fueron inicialmente significativos principalmente en Scopus, con 41 artículos encontrados. Sin embargo, después del filtrado, estos números se redujeron a 24 artículos, de los cuales al analizar el contenido solo nos quedamos con 12 porque la otra mitad ya aparecía en la anterior búsqueda realizada.

Así pues, de la cantidad de resultados iniciales, después del filtrado y análisis del contenido, nos quedamos con 34 artículos escritos en lengua inglesa y 28 en español. Una selección de documentos que posteriormente fueron analizados atendiendo a las variables comentadas y que serán expuestas a lo largo de los siguientes apartados.

\begin{tabular}{|c|c|c|c|c|c|c|c|c|}
\hline \multirow{2}{*}{\multicolumn{2}{|c|}{$\begin{array}{l}\text { Categorías de } \\
\text { búsqueda }\end{array}$}} & \multicolumn{7}{|c|}{ Búsqueda con filtros } \\
\hline & & WOS & SCOPUS & ERIC & $\begin{array}{l}\text { SPORT } \\
\text { DISCUS }\end{array}$ & DIALNET & TOTA & $\begin{array}{c}\text { TOTAL } \\
\text { (con análisis de } \\
\text { contenido) } \\
\end{array}$ \\
\hline $\begin{array}{c}\text { Escuela } \\
\text { rural }\end{array}$ & EF & 1 & 1 & 0 & 1 & 28 & 28 & 28 \\
\hline $\begin{array}{c}\text { Rural } \\
\text { school }\end{array}$ & $\begin{array}{c}\text { Physical } \\
\text { Education }\end{array}$ & 9 & 9 & 7 & 12 & 2 & 22 & 22 \\
\hline $\begin{array}{c}\text { Escuela } \\
\text { rural }\end{array}$ & Deporte & 0 & 0 & 0 & 0 & 19 & 19 & 0 \\
\hline $\begin{array}{c}\text { Rural } \\
\text { school }\end{array}$ & Sport & 3 & 11 & 2 & 8 & 0 & 24 & 12 \\
\hline
\end{tabular}

\section{Publicaciones en función de la edad de la muestra}

Los resultados obtenidos al analizar los documentos escritos en inglés nos reflejan que el 58,62\% de las publicaciones (17 de los 29 trabajos) son estudios con alumnado de edades comprendidas entre los 6 y los 11 años, etapa que en el contexto español equivale a la Educación Primaria (en adelante EP). Por su parte, el 41,38\% de ellos han sido aplicados entre los 12 y 18 años (12 de las 29 publicaciones), equivalentes a la Educación Secundaria.

Por su parte, en los textos escritos en lengua española y que hacen referencia al contexto estatal no vemos esta diferencia entre grupos de edad porque los estudios fueron aplicados en EP, a excepción de uno de ellos. Un trabajo que, sin embargo, se centró en edades comprendidas entre tres y seis años (Martín, 2007).

\section{Publicaciones en función de la localización geográfi-} ca

Respecto a la localización geográfica de los estudios, vemos que se encuentran referencias de diferentes partes del mundo, principalmente provenientes de los Estados Unidos (12 estudios, es decir, el 44,83\%). Le siguen muy de lejos España con tres estudios, así como Sudáfrica y Portugal con dos. En el resto de países solamente aparece un estudio en estas bases de datos (ver figura 2).

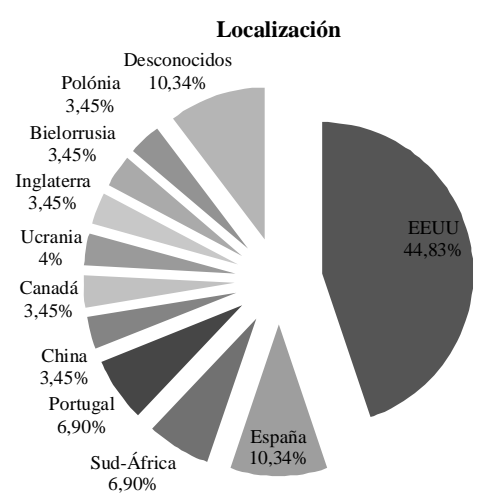

Figura 2. Localización geográfica de los estudios de EF en la escuela rural a nivel internacional

\section{Método de los estudios publicados}

En cuanto al método utilizado para hablar de la temática de la presente revisión, existe un gran predominio de los estudios de carácter cuantitativo en las publicaciones en 
lengua inglesa. Estos estudios han sido llevados a cabo a partir de medidas objetivas $(45,45 \%)$ o de cuestionarios (31,81\%). Es decir, prácticamente dos terceras partes de la totalidad de los estudios son de carácter cuantitativo. Entre el resto de métodos de investigación podríamos diferenciar los estudios cualitativos (9,1\%), los mixtos $(9,1 \%)$ y los ensayos o aproximaciones teóricas también con un 4,54\% del total de los estudios publicados (ver figura 3).

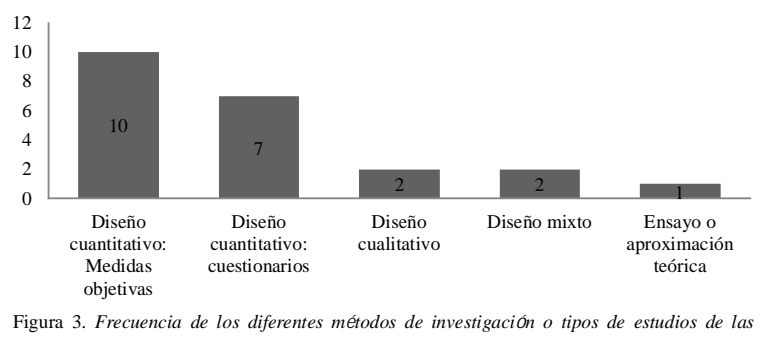

Figura 3. Frecuencia de los diferentes métodos de investigación o tipos de estudios de las publicaciones escritas en lengua inglesa que abordan la EF en la escuela rural.

En contraposición a estos resultados, la mayoría de los estudios publicados en lengua española tienen un carácter de innovación educativa o de descripción de experiencias prácticas (64,28\%). En menor medida, tal y como refleja la figura 4, también se contemplan estudios de carácter cualitativo $(17,86 \%)$ u otros que presentan ensayos o fundamentaciones teóricas (14,28\%). A todo ello, conviene añadir que no se ha encontrado ningún estudio de carácter cuantitativo publicado en lengua española y tan solo un estudio en el que se utilizan métodos mixtos y que, por tanto, representaría el 3,58\% de la totalidad de trabajos publicados.

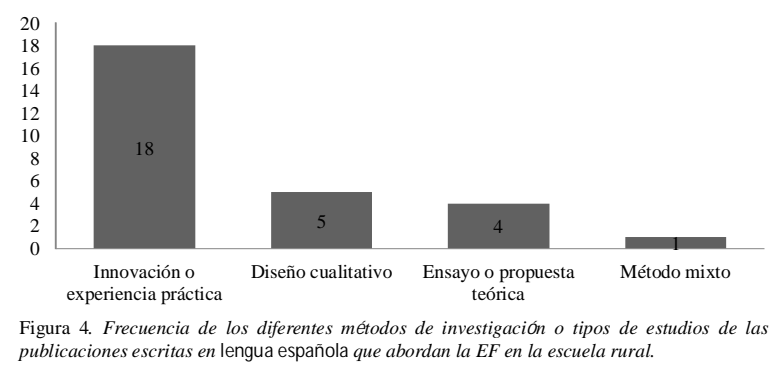

publicaciones escritas en lengua española que abordan la EF en la escuela rural.

\section{Temáticas o contenidos que se estudian}

Respecto a las temáticas que abordan las publicaciones analizadas en lengua inglesa, existe una gran variedad de contenidos, aunque prácticamente la mitad de estas publicaciones (40,90\%) se centran en la actividad física y la salud (ver figura 5). El bloque de juegos y deportes es la segunda temática más estudiada (22,72\%) y le siguen los trabajos que investigan la metodología docente en estas escuelas (13,63\%), la competencia cultural de las y los docentes (9,1\%) y, finalmente, las temáticas sobre la motivación, las habilidades motrices o las preferencias de interacción social son las que menos se estudian, con un 4,54\% del total cada una.

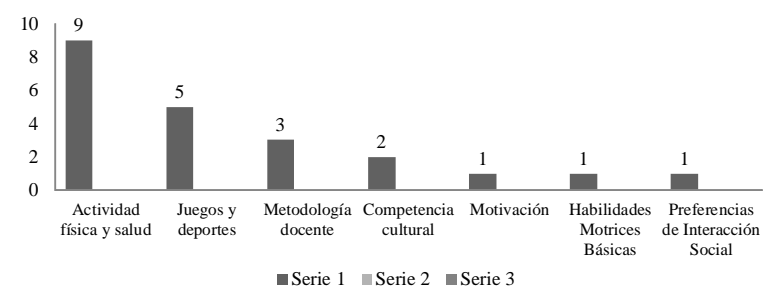

Figura 5. Frecuencia de temáticas o contenidos que se estudian en las publicaciones sobre EF de la escuela rural a nivel internacional.
En los trabajos escritos en lengua española, todas las referencias que trabajan esta temática la abordan desde una visión más pedagógica y educativa del área de EF. Concretamente, a partir de sus características, particularidades, rol de los profesores, usos de los materiales, espacios y posibilidades para llevar a cabo actividades alternativas, como por ejemplo, el medio natural. Por tanto, no se observa esta distinción tan clara entre tendencias no necesariamente educativas, sino un enfoque más unánime centrado en todo aquello que engloba el área de EF en un contexto particular como el rural.

\section{Discusión}

A la hora de discutir estos resultados vemos en primer lugar la mayor parte de los estudios se centran en la mejora y desarrollo de la salud en jóvenes nacidos en entornos rurales, considerando de partida que estos contextos cuentan con menores posibilidades y recursos (Cluss et al., 2016; Cornish, Askelson \& Golembiewski, 2016; Kubayi \& Surujlal, 2014). De este modo, vinculan el entorno rural a los problemas derivados de la falta de actividad física y salud como puede ser la obesidad infantil (Newton Jr. et al., 2011) o el sedentarismo (Machado-Rodrigues et al., 2016). En esta línea, se plantean estudios del índice de masa corporal en poblaciones de riesgo (Eichner et al., 2016) o se desarrollan programas de actividad física para personas con necesidades médicas especiales para mejorar la movilidad y evitar la morbilidad, entre otros (Tymochko-Voloshyn et al., 2015). Un análisis que, como vemos, se limita a asociar el entorno rural a un espacio desfavorecido sin entrar en sus aportaciones y/ o limitaciones educativas. Siguiendo con esta línea, se observan actuaciones destinadas a prevenir adicciones u otras problemáticas. Por ejemplo, la aplicación de programas destinados a la prevención de alcohol (Werch et al., 2000) y de otras drogas (Zavela et al., 2004), aproximaciones que analizan los comportamientos sexuales de riesgo entre adolescentes escolares rurales (Awotidebe et al., 2014) e incluso estudios en los que se vincula la mayor aparición de ansiedad y trastornos neuróticos en las zonas rurales a causa de los conflictos familiares (Krauss et al., 2012). Ejemplos que, de nuevo, dibujan una concepción del entorno rural centrada en las problemáticas sociales relativas a temas de salud pública, hábitos y conductas de riesgo, etc.

Desde un posicionamiento similar al anterior, pero más centrado en el terreno pedagógico, aunque de carácter extraescolar, surgen diversos estudios para tratar de mejorar la actividad física y recreativa de los entornos rurales. Se han remarcado diversos trabajos en los que se plantean programas de actividad para el tiempo libre fuera del centro (Schlatter, Shahrer \& Pogue, 2003) o actividades para cubrir el tiempo escolar en el que no se trabajan contenidos curriculares para mejorar la salud del alumnado (Bershwinger \& Brusseau, 2013; Silva, et al., 2015). A pesar de un enfoque más educativo, se mantiene en estos estudios la necesidad de plantearse qué tipo de concepción social existe sobre los entornos rurales. Y es que vemos que en los países en los que mayoritariamente ha sido estudiado el contexto rural, este parte de una imagen menospreciada, empobrecida o marginada (Feu, 2004; López-Pastor, 2008). Una visión que difiere 
de la situación actual de algunos países occidentales en los que las escuelas rurales cuentan con muchas más posibilidades y recursos (Domingo, 2014).

Por otra parte, se observan publicaciones que se centran en el estudio de determinados contenidos o habilidades trabajadas en las clases de EF de las escuelas rurales. Sobre este aspecto, contemplamos referencias al trabajo de las artes marciales (Bai, 2017), la mejora del salto horizontal (Weimar, Martin \& Wall, 2011), la promoción de deportes como el atletismo en zonas con pocos recursos (Schneider et al., 2017), así como determinados juegos culturales que pueden ser utilizados para promover la inclusión social en entornos desfavorecidos (Roux, Burnett \& Hollander, 2008). Por otra parte, encontramos enfoques que pretenden trabajar la educación deportiva para mejorar la autonomía (Sluder, Buchanan \& Sinelnikov, 2009), estudiar la resistencia y las aptitudes físicas en deportes como el futbol y el atletismo (Murphy et al., 2012) o valorar los efectos del juego y las actividades naturales en la promoción de la salud de los jóvenes (Das \& Chatterjee, 2012). Del mismo modo, vemos experiencias diseñadas para trabajar la estructuración espacio-temporal (Lena y Saludes, 2001) o las posibilidades de la educación deportiva en la escuela rural (Barba, 2007). En resumen, en estas publicaciones predomina el uso de determinados contenidos para mejorar las capacidades físicas y deportivas del alumnado del entorno rural. De este modo, aunque podría existir cierta relación con la idea anterior de mejora de la salud en el alumnado de zonas rurales, este enfoque se constituye a partir de iniciativas educativas que forman parte del área de EF y no de prácticas aisladas de actividad física que pueden formar parte, o no, de las escuelas.

Por otra parte, se distinguen referencias en las que se vincula el aprendizaje en el entorno rural con la participación de las familias y de la comunidad. Sobre todo, a fin de optimizar las prácticas educativas, culturales y deportivas, así como para mejorar el nivel de madurez del alumnado (Klinov \& Blotskiy, 2016), el nivel de actividad física (Loucaides, Plotnikoff, \& Bercovitz, 2007), la relación con la comunidad (Cutforth \& Belansky, 2015) o el propio sistema educativo en conexión con la realidad de las familias (Chermit et al., 2015). Una cuestión que, como proponían López-Pastor (2008) o Ruiz-Omecaña (2008), supone integrar a los diferentes miembros de la comunidad educativa para desarrollar la práctica de la actividad física y deportiva en el ámbito escolar.

Asimismo, se han trabajado experiencias para valorar las diferencias al aplicar estrategias metodológicas de origen cognitivo y/o conductistas, como es el caso de las diferencias entre estilos de enseñanza como el aprendizaje cooperativo y la asignación de tareas, concluyendo que las características de las escuelas rurales se ven favorecidas por los estilos de enseñanza que implican una mayor cognición y participación entre sus miembros (Barba, 2010; Martín\& Barba, 2016). Asimismo, se han llevado a cabo estudios para trabajar las tareas motrices en general desde una visión metodológica centrada en los principios de la Escuela Nueva (Ramírez, Arcila \& Montaño, 2013). En esta línea, conviene mencionar, además, los trabajos de índole teórica que se centran en analizar el funcionamiento, características, particularidades y limitaciones de la EF de la escuela rural, a fin de interpretar las particularidades de este escenario educativo y orientar su práctica. Estas publicaciones analizan el funcionamiento de la EF en este contexto educativo (Boluda \& Fontarnau, 2015; Gracia, 2002; López-Pastor, 2002; LópezPastor, 2008; Uriel, 2002). En ellas se habla de cómo educar en el medio rural, de la necesidad de trabajar la EF de forma distinta a través de espacios y planteamientos didácticos diferentes, con recursos no industrializados o convencionales, a partir de un trabajo más individualizado y conectado con la realidad (Cortés et al., 2002). Una serie de aspectos extraídos en gran medida de estudios de casos que pueden transferirse a realidades similares (Adell, 2004; Barba, 2001; Gómez, 2002). Finalmente, también, tenemos las publicaciones destinadas a analizar cuál es la percepción de los y las docentes cuando llegan a estas escuelas, con qué dificultades se encuentran y cómo pueden actuar para sobrellevarlas y mejorar su práctica (Pedraza-González \& López-Pastor, 2015). En esta línea, se observan análisis que se centran en la percepción que tienen estos sobre la organización escolar y funcionamiento de las escuelas rurales. En particular, destaca en este sentido un estudio en el que se recogen las ventajas evidentes que presenta este entorno, así como aquellos aspectos que quedan por mejorar (Sánchez, Martínez \& Hinojo-Lucena, 2017). Así pues, nos situamos en una temática centrada en analizar la figura del profesorado rural para evidenciar cuáles son las necesidades y/o particularidades que surgen a las y los docentes a la hora de trabajar en un entorno como el rural.

También cabe señalar las experiencias de carácter práctico aportadas por especialistas de EF que trabajan en escuelas rurales. Dentro de estas, se contempla una gran amplitud y diversidad de propuestas centradas en las características o particularidades más beneficiosas y ventajosas de la escuela rural, como la proximidad al medio natural, la ratio reducida, las agrupaciones multigrados, el carácter interdisciplinar predominante, etc. También observamos otros centrados en superar sus dificultades, como la falta de recursos materiales, espaciales y personales. Así pues, se comparten experiencias centradas en aprovechar los espacios que proporciona la escuela rural, permitiendo a la EF ir más allá de los espacios convencionales y trascender a la vida diaria de la comunidad rural (Barba, 2004; Barba, 2006; Santos \& Fernández, 2011). Otros autores, por su parte, ponen énfasis en la necesidad de utilizar materiales alternativos para trabajar en escuelas donde los recursos convencionales son más escasos que en centros de mayores dimensiones. Sobre todo, haciendo uso de materiales reciclados y de desecho aportados por el alumnado u otros de elaboración propia (Dols, 2005).

Por último, algunos autores aportan experiencias en determinados contenidos del área de EF que se ajustan especialmente a estas escuelas, como es el caso de la expresión corporal, debido al potencial de trabajo interdisciplinar que brinda la escuela rural entre la EF y otras áreas curriculares como la Educación Artística (Abad et al., 2017; Echevarría, 2009; Galé, 2017; Ponce de León, Sanz \& Bravo, 2002). Todo ello, contando con la utilización de recursos y estrategias alternativas como puede ser el cine, el teatro de sombras, etc. (Guerrero, 2011; Martín, 2007). 


\section{Conclusiones}

Lo expuesto a lo largo de la presente revisión nos permite llegar a la conclusión de que existen pocos estudios a nivel internacional centrados en el contexto educativo rural. Y es que, como hemos visto a lo largo del análisis, solo existen referencias a la promoción de la actividad física y la salud fuera del ámbito escolar, es decir, a la promoción de actividades en el tiempo libre o al trabajo de determinados contenidos puntuales que han sido aplicados en un contexto rural pero sin la finalidad de analizar o reflexionar sobre la realidad de la asignatura de EF, quedando los aspectos vinculados con el tratamiento propio de la educación formal fuera de las principales bases de datos (WOS, Scopus y ERIC).

Por otro lado, el interés por estas escuelas crece a nivel nacional, donde sí se ha hecho una descripción teórica de todo aquello que engloba el tratamiento del área de EF en estos entornos desde la visión del alumnado y profesorado. Sin embargo, son aportaciones concretas y no actualizadas que pueden ser complementadas con otros estudios en los que se evidencie la evolución de la escuela rural actual, los tipos de escuelas rurales existentes y, en definitiva, las posibilidades educativas de las mismas. Además, como hemos visto, más allá de los estudios teóricos, solo existen aportaciones de experiencias prácticas que, por tanto, no han sido analizadas desde el punto de vista de la investigación. Asimismo, todas ellas han sido llevadas a cabo por escasos autores y a través de trabajos de corta duración y con pocos resultados que muestren las posibilidades de estas escuelas, su relación o diferencias con las escuelas urbanas, etc.

Por tanto, vemos como a nivel internacional el contexto educativo rural parece ser olvidado, menospreciado y vinculado con la precariedad. Por ello, existen estudios que tratan de favorecer este entorno desde el ámbito de la salud y bienestar personal y social. Por su parte, en el ámbito nacional, se incrementa el interés por este contexto educativo, se empiezan a estudiar las particularidades del área de EF pero todo ello se limita a descripciones teóricas de la misma o a aportaciones prácticas sin evidencias científicas. Pese a ello, y teniendo en cuenta las ventajas que se producen al vincular EF y escuela rural, sería necesario empezar a trabajar en mayor medida en diferentes partes del mundo cuáles son las necesidades del área de EF en los contextos educativos rurales, ya que estos presentan particularidades propias, únicas y que podrían generar beneficios pedagógicos.

\section{Referencias}

Abad, C., Campo, M. I., Cortés, M. L. \& Lienas, F. J. (2017). Cine y expresión corporal: Un proyecto interdisciplinar en una escuela rural. Tándem: Didáctica de la EF, (56), 26-33.

Adell, J.A. (2004). La EF en la escuela rural: El caso de la provincia de Huesca. En V. M. López-Pastor, R. Monjas \& A. Fraile (Eds.), Los últimos diez años de la EF escolar: cursos de invierno 2003 (pp. 141-147). Valladolid: Centro Buendía.

Awotidebe, A., Monyeki, A., Phillips, J.\& Lens, W. (2014). The outcomes of a sport-based intervention on risky sexual behaviours among rural school-going adolescents.African Journal for Physical Health Education, Recreation and Dance, 20(4), 1436-1454.
Bai, Z. G. (2017). Wushu Teaching Strategy and Development in Urban and Rural Schools. Eurasia Journal of Mathematics, Science and Technology Education, 13(12), 7791-7797.

Barba, J. J. (2001). Aprendiendo a hacer etnografía durante el Prácticum. Revista interuniversitaria de formación del profesorado, 42, 177-190.

Barba, J. J. (2004). Dónde realizar la EF en la escuela rural. Lecturas: EF y deportes, 79, 5.

Barba, J. J. (2006). Deslizándonos por la nieve en la escuela rural. La actividad física del tiempo libre entra en el aula. Lecturas: EF y deportes, 94, 39.

Barba, J. J. (2007). Posibilidades de la educación deportiva en la escuela rural. Una propuesta a través del voleibol deconstruido. RETOS. Nuevas tendencias en EF, Deporte y Recreación, 11, 46-50.

Barba, J. J. (2010). Diferencias entre el aprendizaje cooperativo y la asignación de tareas en la Escuela Rural. Comparación de dos estudios de caso en una unidad didáctica de acrosport en segundo ciclo de primaria. Retos. Nuevas tendencias en EF, Deporte y Recreación, 18, 14-18.

Bershwinger, T. \& Brusseau, T. A. (2013). The impact of classroom activity breaks on the school-day physical activity of rural children. International journal of exercisescience, 6(2), 134.

Blázquez, D. (2016). Métodos de enseñanza en educación física. Enfoques innovadores para la enseñanza de competencias. Barcelona: Inde.

Boluda, G., \& Fontarnau, F. (2015). EF en la escuela rural. Cuadernos de Pedagogía, 453, 25-29

Bustos, A. (2009). Los grupos multigrado de educación primaria en Andalucía. Tesis doctoral. Granada: Departamento de Didáctica y Organización Escolar de la Universidad de Granada.

Bustos, A. (2011). Enseñar en la escuela rural aprendiendo a hacerlo. Evolución de la identidad profesional en las aulas multigrado. Revista de currículum i formación del profesorado, 11(3)-126.

Chermit, K, Zamalyadinova, O., Khakunova, F., Autleva, A. \&Khatkov, A. (2015). A Pedagogic System of Implementing the Principle of Life and Health Priority of Students at a Rural General Education School. Mediterranean Journal of Social Sciences, 6(5), 44-53.

Cluss, P., Lorigan, D., Kinsky, S., Nikolajski, C., McDermott, A. \& Bhat, K. B. (2016). School-Based Health Promotion Initiative Increases Children's Physical Activity.American Journal of Health Education, 47(6), 343-354.

Cornish, D., Askelson, N. \& Golembiewski, E. (2016). «Reforms Looked Really Good on Paper»: Rural Food Service Responses to the Healthy. Journal of School Health, 86(2), 113-120.

Cortés, N., Gutierrez, C., Lena, C., Martínez, S., Saludes, P., \& Sandín, M. (2002). Una EF diferente para una escuela diferente. La escuela rural. Tándem: Didáctica de la EF, 3(9), 7-19.

Cutforth, N. \& Belansky, E. S. (2015). A community-engaged approach to translating research into practice: A physical education story. Progress in community health partnerships: research, education, and action, 9(4), 571-582.

Das, P., \& Chatterjee, P. (2012). Urban-rural Contrasts in the Cardiorespiratory Fitness of Youngster Footballers in West Bengal, India. International Journal of Applied Sports Sciences, 8(2), 797-805.

Devís-Devís, J. (2018). Los discursos sobre las funciones de la educación física escolar. Continuidades, discontinuidades y retos. Colección Lecciones Magistrales, Publicacions de la Universitat de València, Valencia.

Dols, J. (2005). Reciclaje y materiales para la EF en la escuela rural. Lecturas: EF y deportes, 87, 18.

Domingo, L. (2014). Contribucions pedagògiques de l'escola rural. La inclusió a les aules multigrau: un estudi de cas. Tesis docto- 
ral. Barcelona: Departament de Pedagogia de la Universitat de Vic.

Echevarría, M. C. (2009). Experiencias interdisciplinares en una escuela rural. La Peonza: Revista de EF para la paz, 4, 66-69.

Eichner, J. E., Folorunso, O. A., \& Moore, W. E. (2016). A Physical Activity Intervention and Changes in Body Mass Index at a Middle School With a Large American Indian Population, Oklahoma, 2004-2009. Preventing Chronic Disease, 13, E163.

Feu, J. (2004). La escuela rural en España: apuntes sobre las potencialidades pedagógicas, relacionales y humanas de la misma. Revista Digital eRural, Educación, cultura y desarrollo rural, 2(3), 1-13.

Feu, J. (2004). Les escolesrurals, una realitat en contínuatransformació que augura un futur esperançador. Revista de Girona, 224, 74-78.

Galé, C. (2017). Lataball, una alternativa en la escuela rural. Tándem: Didáctica de la EF, 58, 59-64.

García-Puchades, W. \& Chiva-Bartoll, O. (2018). El juego como proceso de subjetivación en EF y su justificación curricular, Cultura, Ciencia y Deporte, 13, 147-156.

Gómez, C. (2002). La EF en escuelas en el medio rural, una experiencia personal. Tándem: Didáctica De La EF, 3(9), 20-28.

Gracia, F. (2002). La EF en la escuela rural. Tándem: Didáctica De La EF, 3(9), 48-58.

Guerrero, M. D. C. (2011). «Nos expresamos»: una sesión de EF en una escuela unitaria. EmásF: revista digital de EF, 9, 16-26.

Hirst, P.H. (1974). Knowledge and the Curriculum. London: Routledge \& Kegan Paul.

Hirst, P.H.\& Peters, R. S. (1970). The Logic of Education. London: Routledge \& Kegan Paul.

Kirk, D. (2010). Physical Education Futures. London: Routledge.

Klinov, V.\&Blotskiy, S. (2016). Forming senior school students'values of leisure at rural schools in the course of physical education. Science and Education, (8), 79. Epub.

Krauss, H., Buraczynska-Andrzejewska, B., Piatek, J., Sosnowski, P., Mikrut, K., Glowacki, M.\&Zwolinski, J. (2012). Occurrence of neurotic and anxiety disorders in rural schoolchildren and the role of physical exercise as a method to support their treatment.Annals of agricultural and environmental medicine, 19(3). Epub.

Kubayi, N.A.\&Surujlal, J. (2014). Perceived Benefits of and Barriers to Exercise Participation among Secondary School Students. Mediterranean Journal of Social Sciences, 5(20), 121-126.

Lena, C. \&Saludes, P. (2001). EF y Escuela Rural: una UD en torno a la estructuración espacio-temporal. Revista de EF: Renovar la teoría y práctica, 81, 31-38.

López-Pastor, V. M. (2002). Recursos, experiencias y posibilidades para el desarrollo de la EF en la escuela rural. Tándem: Didáctica de la EF, 9, 72-90.

López-Pastor, V. M. (2008). La EF en la escuela rural: singularidades, implicaciones y alternativas en la práctica pedagógica. Contextos educativos: Revista de educación, 11, 249-251.

Loucaides, C. A., Plotnikoff, R. C., \&Bercovitz, K. (2007).Differences in the correlates of physical activity between urban and rural Canadian youth.Journal of School Health, 77(4), 164-170.

Martín, M. I. (2007). Teatro de sombras y festival de navidad: una experiencia en educación infantil en la escuela rural:: ¡ Luz..., pantalla.... y acción!. La Peonza: Revista de EF para la paz, 2, 25-32.

Martín, G.\& Barba, J. J. (2016). La Transformación de una docente y el aula a través de una experiencia centrada en la socialización.Ágora para la EF y el deporte, 18(3), 289-304.

Murphy, A., Kaufman, M. S., Molton, I., Coppel, D. B., Benson, J.\& Herring, S. A. (2012). Concussion evaluation methods among Washington State high school football coaches and athletic trainers. $P M \& R, 4(6), 419-426$.

Newton Jr, R., Thomson, J., Rau, K., Ragusa, S., Sample, A., Singleton, N. \& Williamson, D. (2011). Psychometric characteristics of process evaluation measures for a rural schoolbased childhood obesity prevention study: Louisiana Health. American journal of healthpromotion, 25(6), 417-421.

Pedraza, M. A., \& López-Pastor, V. M. (2015). Investigaciónacción, desarrollo profesional del profesorado de EF y escuela rural. Revista Internacional de Medicina y Ciencias de la Actividad Física y del Deporte/International Journal of Medicine and Science of PhysicalActivity and Sport, 15(57), 1-16.

Ponce de León, A., Sanz, E.\& Bravo, E. (2002). La EF en los colegios rurales agrupados. Tándem: Didáctica De La EF,3(9), 59-71.

Ramírez, Arcila \& Montaño (2013). Tareas motrices. Apuesta comprensiva para su diseño metodológico en Escuela Nueva. EF y Deporte, 32(1), 1277-1285.

Roux, C. J., Burnett, C.\& Hollander, W. J. (2008).Curriculum enrichment through indigenous Zulu games.South African Journal for Research in Sport, Physical Education and Recreation, 30(1), 89-103.

Ruiz-Omeñaca, J. V. (2008). EF para la escuela rural: singularidades, implicaciones y alternativas en la práctica pedagógica. INDE.

Santamaría, R. (1996). La escuela rural entre 1970 y 1990. Zona del río Villahermosa. Tesis doctoral. Castellón: Departamento de Educación de la Universitat Jaume I de Castelló.

Santos, M. L., \& Fernández, L. (2011). Las actividades en el medio natural en la EF de la escuela rural. Un estudio de caso. Profesorado. Revista de Currículum y Formación de Profesorado, 15(2), 219-233.

Schlatter, B. E., Schahrer, S.\& Pogue, P. (2003). After-school recreation in rural settings: A successful partnership. Journal of Physical Education, Recreation \& Dance, 74(4), 24-26.

Schneider, K., Meeteer, W., Nolan, J. A., \& Campbell, H. D. (2017). Health care in high school athletics in West Virginia.Rural and remote health, 17(3879). Epub.

Sena-Gomes, I. \& De Oliveira-Caminha, I. (2014). Guia para estudos de revisão sistemática: uma opção metodológica para as Ciências do Movimento Humano. Movimento, 20(1), 395-411.

Silva, P., Sousa, M., Sá, C., Ribeiro, J., \& Mota, J. (2015). Physical activity in high school during 'free-time'periods.European Physical Education Review, 21(2), 135-148.

Sluder, B. J., Buchanan, A. M., \&Sinelnikov, O. A. (2009). Using sport education to teach an autonomy-supportive fitness curriculum.Journal of Physical Education, Recreation \& Dance, 80(5), 20-28.

Tymochko-Voloshyn, R., Trach, V., Boretsky, Y.\&Dyka, M. (2017). Correction of the functional state of 5-9-grade students at rural schools selected for special medical groups due to articular manifestations of connective tissue dysplasia in Ukraine. Journal of Physical Education and Sport, 17(2), 568.

Uriel, J. R. (2002). EF en el medio rural. Tándem: Didáctica de la $E F, 3(9), 29-47$.

Weimar, W. H., Martin, E. H.\& Wall, S. J. (2011). Kindergarten students' qualitative responses to different instructional strategies during the horizontal jump.Physical Education \& Sport Pedagogy, 16(3), 213-222.

Werch, C. E., Carlson, J. M., Pappas, D. M., Edgemon, P. \&Diclemente, C. C. (2000).Effects of a brief alcohol preventive intervention for youth attending school sports physical examinations.Substance use \& misuse, 35(3), 421-432.

Zavela, K., Battistich, V., Gosselink, C. \& Dean, B. (2004). Say Yes First: Follow up of a five-year rural drug prevention program. Journal of drug education, 34(1), 73-88. 